\title{
DETERMINACIÓN DE LA FRECUENCIA, CAUSAS Y EVITABILIDAD DE LOS EVENTOS ADVERSOS EN EL SERVICIO DE PEDIATRÍA DE UN IPS QUINDIO 2014
}

\author{
DETERMINATION OF FREQUENCY, CAUSES AND PREVENTABILITY \\ OF ADVERSE EVENTS IN THE PEDIATRIC WARD A IPS QUINDIO 2014
}

María Camila Rendón Román ${ }^{1}$, Smyd Vanneza Restrepo franco ${ }^{2}$, Jheimy Jackeline García Castañeda ${ }^{3}$

1. Enfermera. Especialista en Gerencia de La calidad y auditoria en Salud Coordinadora de la unidad renal.

2. Enfermera. Especialista en Gerencia de la calidad y Auditoria en Salud Coordinadora de programas en secretaria de Calarcá.

3. Enfermera. Especialista en gerencia de la calidad y auditoria en salud. Magister en Salud Pública Docente Programa de enfermería Facultad de Ciencias de la Salud Universidad del Quindío. Integrante del Grupo GIAPS

Recibido: 4 Diciembre de 2015

Aceptado: 15 Febrero de 2016

*Correspondencia del autor: Jheimy Jackeline Garcia Castañeda. E-mail: jheimyjacke@gmail.com

\begin{abstract}
RESUMEN
La seguridad del paciente involucra una responsabilidad legal y ética del prestador de servicios de salud, en el ejercicio de esta profesión, de forma competente, segura, con autodeterminación y autorregulación de sus acciones. (1) objetivo: Determinar la frecuencia, causas y evitabilidad de los eventos adversos en el servicio de pediatría de una IPS Quindío 2014 metodología: se desarrolló un estudio cuantitativo, retrospectivo de corte transversal, con un muestreo tipo censo, Se realizó una análisis con el SPSS V17; Univariado: en el cual se identificaron las características sociodemográficas, los porcentajes de los eventos adversos, sus causas y Análisis bivariado: para hacer comparaciones entre grupos, se realizaron tablas de 2 por 2, para evaluar las variables categóricas se realizó la prueba de chi2 y el test exacto de Fischer, con un nivel alfa de 0,05 que determinó la significancias estadística. Conclusiones: Se encontró que los eventos adversos en el servicio de pediatría fueron más frecuentes en lactantes $60 \%$ y preescolares de género femenino $40 \%$, el $64,3 \%$ Los eventos adversos presentes en este servicio se atribuyen a causas relacionadas con el cuidado directo de los pacientes; un $85,7 \%$ de los casos demuestra que existen fallas en las barreras humanas y administrativas que predisponen de manera directa a la ocurrencia de eventos adversos. El 73.3\% de la población pertenece al régimen subsidiado de los eventos adversos presentados un $64,3 \%$, El $73.3 \%$ de los eventos adversos que ocurrieron en el periodo de tiempo evaluado tienen causas prevenibles.
\end{abstract}

Palabras claves: Evento adverso, Pediatría 
Revista de Investigaciones - Universidad del Quindío

\begin{abstract}
Patient safety involves legal and ethical responsibility of the health care provider, in order to perform this profession in a competitive and safe way, with self-determination and self-regulation of its actions. (1) Objective: to determine the frequency, causes, and avoidability of adverse events in the pediatrics department of a HSP in the department of Quindío, in 2014. Methodology: a quantitative, retrospective, cross-sectional study was performed with a census sampling method; an analysis with SPSS V17 was performed, Univariate: in which the sociodemographic characteristics, the percentages of adverse events, their causes and bivariate analysis were identified: to make comparisons between groups, 2 by 2 tables were made; to evaluate categorical variables Chi2 test and Fischer's exact test were performed with an alpha level of 0.05 , which determined the statistical significance. Conclusions: It was found that adverse events in the pediatrics department were more common in infants $(60 \%)$ and female preschool children $(40 \%), 64.3 \%$ of adverse events occurring in this service are attributed to causes related to direct patients care; $85.7 \%$ of the cases shows flaws in human and administrative barriers which predispose occurrence of adverse events directly. $73.3 \%$ of the population belongs to the subsidized regime of the adverse events presented $64.3 \%$, of the adverse events that occurred in the period of time evaluated are preventable causes.
\end{abstract}

Keywords: Adverse event, Pediatrics

\section{INTRODUCIÓN}

Discernir sobre el tema de las atenciones inseguras o indicios de atención insegura, es complejo, polémico y difícil de estudiar, razón por la cual, se encuentra cierto rechazo y resistencia para su abordaje, aunado al desconocimiento, lo que, en la mayoría de los casos, levanta barreras que no permiten la integralidad en la investigación, restringiendo la generación e implementación de planes de mejora que contribuyan a minimizar los riesgos.

En Colombia, a pesar de que desde el 2006 con el Decreto 1011, el Ministerio de Salud y Protección Social, a través del sistema obligatorio de garantía de calidad, estableció la transversalidad en sus 4 ejes (sistema único de habilitación, acreditación, sistema de información para la calidad y auditoría para el mejoramiento de la calidad), el componente de seguridad que se sustentó, para el año 2008, con la publicación los lineamientos para la implementación de la política de seguridad del paciente para la prestación de los servicios de salud.(2)

Es evidente, la falta aun de cultura del personal de salud frente al proceso de reporte y respectivo análisis de las atenciones inseguras; lo que dificulta la determinación de la frecuencia, causas y evitabilidad de los eventos adversos, que oriente a los actores, involucrados en el sistema de salud, en la toma de decisiones en la definición e implementación de estrategias que posibiliten la minimización de los riesgos derivadas de la prestación de los servicios de salud, gran parte de estas representadas en el esfuerzo individual, colectivo e interno de las instituciones prestadoras de salud. Situación que obstaculiza la determinación de los mismos, en servicios específicos como el de pediatría, máxime si se tiene en cuenta las connotaciones que hacen más vulnerable a la población infantil, situación que se explicita en la institución de segundo nivel, en la que se desarrolló el proyecto.

Actualmente la OMS se ocupa por el desarrollo de una metodología para el estudio de eventos adversos, la seguridad del paciente, y los errores de la atención médica.(3)

El estudio IBEAS: Prevalencia de efectos adversos en hospitales de Latinoamérica, tenía por objeto conocer la prevalencia de efectos adversos en hospitales de Latinoamérica, para obtener una aproximación a la magnitud, trascendencia e impacto de los eventos adversos (EA); identificar áreas, problemas prioritarios de la seguridad del paciente; involucrar mayor número de profesionales del sector salud en la seguridad del paciente e incorporar en las prioridades de estos países objetivos y actividades para mejorar la seguridad del paciente.(4)

La implementación de dicho estudio en Colombia favoreció la creación de una estrategia de vigilancia de eventos adversos desde el año 2006, en busca de identificar situaciones que podrían calificarse como atención insegura, para analizar las causas de su ocu- 
rrencia y generar acciones que hagan la atención segura y con el mínimo de riesgo, en cada una de las instituciones prestadores de servicios de salud (IPS). (5)

Con el estudio IBEAS no se inicia la política de seguridad del paciente de Colombia, pero sí le hace importantes aportes, brindando a las instituciones una metodología que permite evaluar la frecuencia con la cual se presentan los eventos adversos en sus condiciones específicas, y a su vez medir a mediano plazo el impacto de la implementación de la política de seguridad. A nivel de Colombia, el Ministerio de Salud y Protección Social, ha abordado la problemática desde la perspectiva de la calidad, donde el ente territorial de salud, del orden nacional, implementa el Observatorio de Calidad de la Atención en Salud, con el fin de recopilar, analizar y difundir información importante sobre la calidad de la atención en salud.(6)

En el 2004, con la colaboración de ICONTEC, se estableció como prerrequisito necesario para aplicar a la acreditación en salud, que la institución demostrara que desarrollaba procesos de vigilancia de los mencionados eventos.(7)

En España, por ejemplo, en la revista de calidad asistencial, en el año 2011, publicaron un estudio similar llamado Seguridad clínica de los pacientes durante la hospitalización en pediatría, el cual tenía como objetivo: "Describir la incidencia y tipos de eventos adversos (EA) en pacientes en edad pediátrica y su evitabilidad"(8) Allí se analizaron los eventos adversos ocurridos en 41 hospitales de España, en el periodo de 1 año, con la revisión y análisis de 8,407 historias clínicas:

En 24 de estos pacientes, se detectó algún EA, por lo que la incidencia de EA en la edad pediátrica fue de un 3,6\% (IC: 2,19-5,03\%). Por grupos de edad, los menores de 18 meses (lactantes) tuvieron una incidencia de EA de 3,8\%, similar a la del grupo de 18 meses a 6 años (preescolares), mientras que el grupo de 7 a 15 años (escolares) experimentó una tasa de EA algo menor en torno al 2,6\%". (8) La mayoría de estos eventos adversos se encuentran en la clasificación de prevenibles.(8)

Dentro de las conclusiones importantes se encontró que a pesar que en dichas instituciones se tiene implementado política de seguridad con estándares de calidad, aún se encuentran evidencias de atenciones insegura, además de resaltar que el liderazgo que ejerce el personal de salud y personal administrativo, el seguimiento a la aplicación de estándares son indispensables en el control de los riesgos para la seguridad en las instituciones.(4)

Un estudio similar es el de Frecuencia, evitabilidad y consecuencias de los eventos adversos, como su nombre lo indica, su objetivo fue el de determinar dichos acontecimientos ocurridos en los servicios de hospitalización, basándose en las fichas epidemiológicas e historias clínicas de pacientes que presentaron un evento durante el periodo analizado, primer semestre del año 2010. Por su parte, de un total de 472 eventos reportados se observa que La manera más viable de controlar y disminuir la incidencia de eventos adversos en la institución es promoviendo como también culturizando al personal de salud, con temas que aborden la aplicabilidad de normas de seguridad del paciente, reafirmar los conocimientos sobre las diferentes practicas asistenciales que conllevan en mayor medida la aparición de esos incidentes.(9)

Los eventos adversos en pacientes hospitalizados en tres instituciones hospitalarias en Colombia; realizado por Gaitán-Duarte, et al. (2006), Cuyo objetivo giró en torno a la determinación de la incidencia, clasificación, evitabilidad e impacto de los Eventos Adversos (EA), para establecer su importancia como problema en algunos hospitales en Colombia; se tomaron para este, pacientes hospitalizados en 3 instituciones de Colombia “... muestra que al menos el (4,5 $\%$ ) de los pacientes en servicios quirúrgicos, médicos o de ginecobstetricia de las tres instituciones observadas presentaron eventos adversos."(10)

Cabe resaltar que este estudio es la primera aproximación epidemiológica publicada sobre el tema en Colombia y muestra que al menos el $(4,5 \%)$ de los pacientes hospitalizados, en servicios quirúrgicos, médicos o de ginecobstetricia de las tres instituciones observadas presentaron eventos adversos siendo este un porcentaje elevado de eventos, lo cual puede verse relacionado propiamente con el tipo servicios y complejidad de los mismos.

En nuestro país el Sistema de Seguridad Social en Salud es complejo, la implementación de la política de la seguridad del paciente ha permitido que la atención en salud pase de ser complicada o poco confia- 
ble, para convertirse en efectiva, positiva y proactiva, gracias a los controles, seguimientos y evaluaciones de cada uno de los procesos. Estos métodos ahora son más seguros, avanzados; ya que se han incorporado y renovado los equipos tecnológicos y las técnicas que son cada vez más elaboradas. (11)

Para que la atención en salud sea positiva, es preciso que los diferentes actores involucrados adquieran una participación responsable; también, implica una efectiva planeación, seguimiento y evaluación de cada uno de los servicios a prestar; y con estos identificar los riesgos asociados, diseñar e implementar, de manera constante, las barreras de seguridad necesarias para evitar estos accidentes. Cuando se presenta un evento adverso sufre daños tanto el paciente como el profesional de la salud, debido a la falla en la realización de los procesos. (11)

Teniendo en cuenta que la Seguridad del paciente es la ausencia de daños causados por los profesionales de la salud, en el cual se prolonga la hospitalización que, por ende, puede ocasionar discapacidades y pérdidas económicas. Desde la Asamblea Mundial de la Salud, en 2002, se reconoce la seguridad del paciente como un problema serio de salud pública, especialmente en los países en desarrollo, en los cuales el riesgo de infección intrahospitalaria es mayor. Como respuesta a este problema, la Organización Mundial de la Salud (OMS) en el año 2014, dio a conocer oficialmente la alianza Mundial para la Seguridad del paciente. (12)

De igual forma, Colombia en el año 2006, establece mediante la Resolución 1446, la vigilancia de los eventos adversos y en el 2008 publica los lineamientos de la Política de la seguridad del paciente. Existen estudios previos como el de IBEAS (Prevalencia de efectos adversos en hospitales de Latinoamérica), que tiene como finalidad medir los eventos adversos presentados durante la estancia intrahospitalaria. (4)

Por otra parte el protocolo de Londres es una versión revisada y actualizada de un documento previo conocido como Protocolo para investigar como también analizar de incidentes clínicos. (13) que constituye una guía práctica para los administradores de riesgo, igualmente para otros profesionales interesados en este tema El cual se desarrolló teniendo en cuenta la experiencia en investigación de accidentes, tanto en el sector de la salud como de otras industrias que han avanzado enormemente en su prevención. Su propó- sito es facilitar la investigación clara y objetiva de los incidentes clínicos, lo cual implica ir mucho más allá de simplemente identificar la falla o de establecer quién tuvo la culpa.

En razón a lo expuesto y dada la necesidad de verificar la presencia y abordaje de los eventos adversos en el servicio de Pediatría.:

\section{OBJETIVOS}

\section{Objetivo general}

Determinar la frecuencia, causas y evitabilidad de los eventos adversos en el servicio de pediatría de un hospital de segundo nivel de atención, durante el primer semestre del año 2014

\section{METODOLOGIA \\ Tipo de estudio}

La presente investigación corresponde a un estudio cuantitativo; por ello se realizará un estudio descriptivo retrospectivo de corte transversal, en el cual se analizará la información obtenida de los eventos adversos registrados durante el tiempo comprendido entre enero y junio de 2014, en una IPS del departamento del Quindío. (14) El estudio es de tipo descriptivo de cohorte transversal es transversal; para lo cual se va a realizar descripción y documentación acerca de los eventos adversos presentados, con el fin de conocer la trazabilidad de los mismos; causa, frecuencia evitabilidad como también las consecuencias de los mismos. (13)

Los datos retrospectivos se describen como estudios que se realizan a partir de fuentes secundarias, tales como: archivos, historias clínicas de hospitales, cementerios, registro civil, informes de laboratorio, entre otras. En muchos casos la información ha sido captada por otros con anterioridad. (13) El porcentaje global de eventos adversos en la IPS es de 4,2 sobre el total de 353 pacientes pediátricos en el período analizados.

\section{Población}

Corresponde a un total de 15 casos de atenciones inseguras y eventos adversos presentados en el servicio de Pediatría, de una IPS, durante el período transcurrido entre enero y junio de 2014.

\section{Muestra de estudio}

Se realizara un muestreo tipo censo en el cual se estudian todos los elementos de la población objeto. 


\section{Metodología para la selección de los sujetos de es- tudio}

No aplica, en razón de que se tomará el 100\% de los registros de un instrumento que recopila la información relacionada con atenciones inseguras y eventos adversos en un período de tiempo definido. Es importante anotar que los datos fueron suministrados por la IPS, en cuanto a los RIPS y la base de los eventos adversos del servicio de pediatría correspondiente a los meses de enero a junio del año 2014.

\section{Instrumentos, técnicas y métodos para la recolec- ción de datos}

El trabajo inicia con una fase exploratoria, con el objetivo de documentar la realidad que se analizaron, Como parte del proceso, se revisaron los RIPS y la base de datos de reporte de eventos adversos ocurridos en el servicio de pediatría durante el periodo enero a junio de 2014, a la cual se le aplicará un instrumento que incluye variables sociodemográficas, como también las características asociadas al evento adverso.

\section{Instrumento}

Se diseñó un instrumento en Excel y se aplicó un formato de compilación que recoge las variables definidas para este estudio, las cuales son: la fecha del evento adverso, descripción del evento, genero (sexo) del paciente, edad, régimen de salud, causa, factores contributivos, por ultimo barreras y defensas.

\section{Técnica}

Observación, revisión y análisis documental de las variables en su contexto.

\section{Marco ético}

La investigación se realizó con el aval del Gerente de la IPS. No se contó con el dictamen de aprobación del Comité de ética, por no tratarse de una investigación de tipo biomédico y no realizarse directamente con personas. Con base en lo estipulado en el Artículo 11 de la resolución 008430 de 1993 se trata de una investigación sin riesgo, dado que no se interviene o modifica intencionadamente las variables biológicas, fisiológicas, psicológicas o sociales de las personas y por lo tanto, se utilizó un consentimiento institucional. Igualmente, en el desarrollo de esta investigación, se dio cumplimiento a los principios básicos éticos, valores, deberes y obligaciones, con el fin de respetar entre otros derechos, la confidencialidad de la empresa, en cumplimiento del objetivo descrito en este estudio.
Sistematización de datos y análisis de la información Se sistematizó la información en una base de datos en Excel, con tablas dinámicas para su posterior análisis estadístico, cruce de variables y la construcción de cuadros y gráficos. Para el análisis de los datos se utilizó la Base de Datos SPSS(17), se realizó un análisis univariado y bivariado:

- Análisis univariado: en el cual se identificaron las características sociodemográficas y los porcentajes de los eventos adversos y sus causas.

- Análisis bivariado: para hacer comparaciones entre grupos, se realizaron tablas de 2 por 2.

- En el análisis bivariado se cruzaron los tipos de eventos y las causas de los mismos, realizados con todas las variables sociodemográficas y con la clasificación de los eventos. Para evaluar las variables categóricas se realizó la prueba de $\mathrm{chi}^{2} \mathrm{y}$ el test exacto de Fischer, con un nivel alfa de 0,05 que determinó la significancias estadística.

\section{RESULTADOS}

TABLA. 1: Prevalencia de factores de Relacionados con el evento adverso, en el servicio de Pediatría de una IPS del Quindío 2014

\begin{tabular}{|c|c|c|c|c|}
\hline Variable & Resultado & n & $\begin{array}{c}\text { Fre- } \\
\text { cuencia }\end{array}$ & $\begin{array}{l}\text { Prevalen- } \\
\text { cia }\end{array}$ \\
\hline $\begin{array}{l}\text { Ocurrencia del } \\
\text { Evento }\end{array}$ & Mes de mayo & 15 & 4,95 & $33 \%$ \\
\hline $\begin{array}{l}\text { Causa del } \\
\text { Evento Ad- } \\
\text { verso }\end{array}$ & $\begin{array}{l}\text { Caídas del } \\
\text { Paciente }\end{array}$ & 15 & 6 & $40 \%$ \\
\hline Edad & $\begin{array}{l}\text { Lactantes de } \\
0 \text { a } 18 \text { meses }\end{array}$ & 15 & 9 & $60 \%$ \\
\hline $\begin{array}{l}\text { Régimen de } \\
\text { Salud }\end{array}$ & $\begin{array}{l}\text { Régimen } \\
\text { Subsidiado }\end{array}$ & 15 & 10,9 & $73,3 \%$ \\
\hline $\begin{array}{l}\text { Causas del } \\
\text { Evento }\end{array}$ & $\begin{array}{l}\text { Evento Preve- } \\
\text { nible }\end{array}$ & 15 & 10,9 & $73,3 \%$ \\
\hline $\begin{array}{l}\text { Factores Con- } \\
\text { tributivos }\end{array}$ & $\begin{array}{l}\text { Asociación } \\
\text { Paciente- } \\
\text { Equipo-Am- } \\
\text { biente }\end{array}$ & 15 & 7 & $46,7 \%$ \\
\hline Barreras & $\begin{array}{l}\text { Humanas-Ad- } \\
\text { ministrativas }\end{array}$ & 15 & 6,9 & $46 \%$ \\
\hline
\end{tabular}

Como muestra la tabla 1 en el mes de mayo se presentaron el 33\% de la ocurrencia de los eventos adversos. Las caídas en el servicio de pediatría ocupan el primer lugar en ocurrencia de eventos adversos con 6 de los 15 casos reportados representando el $40 \%$, seguido por 4 casos de reingresos por la misma causa con un $26 \%, 2$ fallas relacionadas con proceso de laboratorio y 2 de errores u omisiones en la administración de 
medicamentos. El $60 \%$ de los eventos adversos corresponden a lactantes ( 0 y 18 meses), mientras que el $40 \%$ en preescolares (entre 18 meses y 5 años), sin presentarse ningún evento en los niños escolares (5 años a 16 años).E1 $73.3 \%$ de la población pertenece al Régimen de salud subsidiado. E1 73.3\% de los eventos adversos son prevenibles. Los factores contributivos asociado a cada una de las acciones inseguras más típicos son paciente - equipo y ambiente equivalente a un $46,7 \%$.La barrera que se presentan con mayor frecuencia son las humanas - administrativas con un $46 \%$.

\section{ANÁLISIS BIVARIADO}

Se realizó una observación univariado de la frecuencia de los eventos. De otra parte se efectúa un análisis bivariado relacionado con la ocurrencia del evento, causa, edad, régimen de salud, factores contributivos y las barreras de presentación del evento.

A continuación se describen los factores de riesgo que tiene porcentajes relevantes dentro del estudio

Tabla 1: Relación de evento adverso con género (sexo) de la población

\begin{tabular}{|c|c|c|c|c|}
\hline & & \multicolumn{2}{|c|}{ SEXO } & \multirow[b]{2}{*}{ Total } \\
\hline & & $\begin{array}{l}\text { FEME- } \\
\text { NINO }\end{array}$ & $\begin{array}{l}\text { MASCU- } \\
\text { LINO }\end{array}$ & \\
\hline EVENTO 1.00 & Recuento & 5 & 2 & 7 \\
\hline ADVERSO 0 & $\begin{array}{l}\% \text { dentro } \\
\text { de EVEN- } \\
\text { TO AD- } \\
\text { VERSO }\end{array}$ & $71.4 \%$ & $28.6 \%$ & $100.0 \%$ \\
\hline
\end{tabular}

Tabla 3 de contingencia EVENTO ADVERSO * edad evento

\begin{tabular}{|c|c|c|c|c|c|}
\hline & & & $\begin{array}{c}\text { edad } \\
\text { lactante }\end{array}$ & $\begin{array}{c}\text { evento } \\
\text { preescolar }\end{array}$ & Total \\
\hline \multirow[t]{6}{*}{ EVENTO ADVERSO } & \multirow{3}{*}{ fallas administrativas } & Recuento & 2 & 3 & 5 \\
\hline & & $\%$ dentro de EVENTO ADVERSO & $40.0 \%$ & $60.0 \%$ & $100.0 \%$ \\
\hline & & $\%$ dentro de edad evento & $22.2 \%$ & $50.0 \%$ & $33.3 \%$ \\
\hline & \multirow{3}{*}{ fallas en cuidado directo } & Recuento & 7 & 3 & 10 \\
\hline & & $\%$ dentro de EVENTO ADVERSO & $70.0 \%$ & $30.0 \%$ & $100.0 \%$ \\
\hline & & $\%$ dentro de edad evento & $77.8 \%$ & $50.0 \%$ & $66.7 \%$ \\
\hline \multirow[t]{8}{*}{ Total } & & Recuento & 9 & 6 & 15 \\
\hline & & $\%$ dentro de EVENTO ADVERSO & $60.0 \%$ & $40.0 \%$ & $100.0 \%$ \\
\hline & & $\%$ dentro de edad evento & $100.0 \%$ & $100.0 \%$ & $100.0 \%$ \\
\hline & & $\%$ dentro de EVENTO ADVERSO & $71.4 \%$ & $28.6 \%$ & $100.0 \%$ \\
\hline & & $\%$ dentro de SEXO & $55.6 \%$ & $33.3 \%$ & $46.7 \%$ \\
\hline & 2.00 & Recuento & 4 & 4 & 8 \\
\hline & & $\%$ dentro de EVENTO ADVERSO & $50.0 \%$ & $50.0 \%$ & $100.0 \%$ \\
\hline & & $\%$ dentro de SEXO & $44.4 \%$ & $66.7 \%$ & $53.3 \%$ \\
\hline \multirow[t]{3}{*}{ Total } & & Recuento & 9 & 6 & 15 \\
\hline & & $\%$ dentro de EVENTO ADVERSO & $60.0 \%$ & $40.0 \%$ & $100.0 \%$ \\
\hline & & $\%$ dentro de SEXO & $100.0 \%$ & $100.0 \%$ & $100.0 \%$ \\
\hline
\end{tabular}

En cuanto a la relación entre el evento adverso y el sexo se observa que el $55,6 \%$ de la población afectada por sucesos negativos en la prestación de los servicios de salud son mujeres (Tabla \#2).

En cuanto a la relación entre el evento adverso y la La relación entre el evento adverso y el régimen de edad se observa que el 77,8\% de la población afectada son lactantes y preescolares (de 0 a 5 años) como también presentaron fallas en el cuidado directo (Tabla \#3) 
salud se observa que el 64,3\% de la población tiene régimen subsidiado y/o vinculado, fueron afectadas por las fallas en el cuidado directo. (Tabla \#4).

Tabla 4 de contingencia EVENTO ADVERSO * Régimen

\begin{tabular}{|c|c|c|c|c|c|}
\hline & & & \multicolumn{2}{|c|}{ Régimen } & \multirow[b]{2}{*}{ Total } \\
\hline & & & $\begin{array}{l}\text { subsidiado y } \\
\text { vinculado }\end{array}$ & Contributivo & \\
\hline \multirow[t]{6}{*}{ EVENTO ADVERSO } & \multirow{4}{*}{ fallas administrativas } & Recuento & 5 & 0 & 5 \\
\hline & & $\%$ dentro de EVENTO ADVERSO & $100.0 \%$ & $.0 \%$ & $100.0 \%$ \\
\hline & & $\%$ dentro de Régimen & $35.7 \%$ & $.0 \%$ & $33.3 \%$ \\
\hline & & Recuento & 9 & 1 & 10 \\
\hline & \multirow[t]{2}{*}{$\begin{array}{l}\text { fallas en cuidado } \\
\text { directo }\end{array}$} & $\%$ dentro de EVENTO ADVERSO & $90.0 \%$ & $10.0 \%$ & $100.0 \%$ \\
\hline & & $\%$ dentro de Régimen & $64.3 \%$ & $100.0 \%$ & $66.7 \%$ \\
\hline \multirow[t]{3}{*}{ Total } & & Recuento & 14 & 1 & 15 \\
\hline & & $\%$ dentro de EVENTO ADVERSO & $93.3 \%$ & $6.7 \%$ & $100.0 \%$ \\
\hline & & $\%$ dentro de Régimen & $100.0 \%$ & $100.0 \%$ & $100.0 \%$ \\
\hline
\end{tabular}

En cuanto a la relación entre el evento adverso y las barreras por las que se puede ocasionar se observa que el $85,7 \%$ de la población involucran barreras humanas y administrativas fueron también afectados por las fallas en el cuidado directo (Tabla \#5).

Tabla 5 de contingencia EVENTO ADVERSO * Barreras del Evento

\begin{tabular}{|c|c|c|c|c|c|}
\hline & & & \multicolumn{2}{|c|}{ Barreras del Evento } & \multirow[b]{2}{*}{ Total } \\
\hline & & & $\begin{array}{l}\text { humanas-ad- } \\
\text { ministrativas }\end{array}$ & $\begin{array}{l}\text { humanas } \\
\text { físicas }\end{array}$ & \\
\hline \multirow[t]{7}{*}{ EVENTO ADVERSO } & \multirow{3}{*}{$\begin{array}{l}\text { fallas administra- } \\
\text { tivas }\end{array}$} & Recuento & 1 & 4 & 5 \\
\hline & & $\begin{array}{l}\% \text { dentro de EVENTO AD- } \\
\text { VERSO }\end{array}$ & $20.0 \%$ & $80.0 \%$ & $100.0 \%$ \\
\hline & & $\begin{array}{l}\% \text { dentro de Barreras del } \\
\text { Evento }\end{array}$ & $14.3 \%$ & $50.0 \%$ & $33.3 \%$ \\
\hline & \multirow{4}{*}{$\begin{array}{l}\text { fallas en cuidado } \\
\text { directo }\end{array}$} & Recuento & 6 & 4 & 10 \\
\hline & & $\begin{array}{l}\% \text { dentro de EVENTO AD- } \\
\text { VERSO }\end{array}$ & $60.0 \%$ & $40.0 \%$ & $100.0 \%$ \\
\hline & & $\begin{array}{l}\% \text { dentro de Barreras del } \\
\text { Evento }\end{array}$ & $85.7 \%$ & $50.0 \%$ & $66.7 \%$ \\
\hline & & Recuento & 7 & 8 & 15 \\
\hline Total & & $\begin{array}{l}\% \text { dentro de EVENTO AD- } \\
\text { VERSO }\end{array}$ & $46.7 \%$ & $53.3 \%$ & $100.0 \%$ \\
\hline
\end{tabular}

En cuanto a la relación entre el evento adverso y los factores se observa que el $70 \%$ de la población que presentan barreras en el paciente - equipo tuvieron fallas en el cuidado directo (Tabla \#6)

\section{Discusión}

La relación entre el evento adverso y la edad se observa que el $77,8 \%$ de la población afectada es lac- tante (de 0 a 5 años), como también presentaron fallas en el cuidado directo. Este resultado es similar a lo encontrado en el estudio Seguridad clínica de los pacientes durante la hospitalización en pediatría, se identificaron 24 pacientes, de los cuales el 3,8\% de los eventos adversos se presentaron en lactantes $(0-18$ meses).(15) 
Tabla 6 de contingencia EVENTO ADVERSO * factores evento

\begin{tabular}{|c|c|c|c|c|c|}
\hline & & & \multicolumn{2}{|c|}{ factores evento } & \multirow[b]{2}{*}{ Total } \\
\hline & & & $\begin{array}{l}\text { paciente- } \\
\text { equipo }\end{array}$ & $\begin{array}{l}\text { tarea-indivi- } \\
\text { duo-paciente }\end{array}$ & \\
\hline \multirow{6}{*}{$\begin{array}{l}\text { EVENTO AD- } \\
\text { VERSO }\end{array}$} & fallas administrativas & Recuento & 3 & 2 & 5 \\
\hline & & $\%$ dentro de EVENTO ADVERSO & $60.0 \%$ & $40.0 \%$ & $100.0 \%$ \\
\hline & & $\%$ dentro de factores evento & $30.0 \%$ & $40.0 \%$ & $33.3 \%$ \\
\hline & fallas en cuidado & Recuento & 7 & 3 & 10 \\
\hline & directo & $\%$ dentro de EVENTO ADVERSO & $70.0 \%$ & $30.0 \%$ & $100.0 \%$ \\
\hline & & $\%$ dentro de factores evento & $70.0 \%$ & $60.0 \%$ & $66.7 \%$ \\
\hline \multirow[t]{3}{*}{ Total } & & Recuento & 10 & 5 & 15 \\
\hline & & $\%$ dentro de EVENTO ADVERSO & $66.7 \%$ & $33.3 \%$ & $100.0 \%$ \\
\hline & & $\%$ dentro de factores evento & $100.0 \%$ & $100.0 \%$ & $100.0 \%$ \\
\hline
\end{tabular}

Igualmente en un hospital pediátrico de la ciudad de Santiago, Chile; su objetivo fue identificar los Eventos Adversos presentes en la atención de niños con enfermedades respiratorias crónicas. (16) El 82,6 \% de la población se encuentra en el intervalo de edad de 1 mes a 5 años con mayor ocurrencia de errores en la prestación del servicio de salud. Por otra parte, el estudio de Efectos adversos asociados a la vacunación PAI en niños menores de 2 años en Medellín reportados entre 2008-2009;(17) tiene similitud con la presente investigación planteada, debido al rango de edad de la población objeto.

Este fue un estudio observacional descriptivo, donde se determinaron los principales efectos adversos generados por la vacunación PAI en niños menores de 2 años. La información se obtuvo de la base de datos SIVIGILA, sobre niños que fueron reportados con efectos adversos entre 2008 y 2009. Se analizaron los datos de variables cualitativas por medio de gráficas, tablas y proporciones, y las variables cuantitativas por medio de medidas descriptivas. Como resultados se encontró que la edad en la que se presentaron mayor número de efectos adversos fue entre 12 y 14 meses de vida, seguido por los primeros 4 meses de vida.

En el mismo se concluyó que la edad pico de presentación de los efectos adversos post vacúnales fue de un año de edad, también que la gran mayoría de estos accidentes se presentaron durante el primer día después de la aplicación. Los efectos de los sucesos más comunes fueron el dolor local y eritema, las vacunas más relacionadas con los efectos adversos fueron la Pentavalente como también el DPT, que juntas conformaron más del 70\% de los efectos adversos reportados. El $50 \%$ de los pacientes se recuperaron sin secuelas.

Toda organización, como un sistema en sí mismo, requiere de una constante evaluación de sus procesos con el fin de mejorar la calidad de éstos. Una institución de salud debe continuamente buscar las herramientas necesarias para otorgar la mejor atención posible a sus usuarios, destinando los recursos humanos como también los materiales necesarios para que la atención sea total, precisa y tan efectiva como el estado actual del conocimiento lo permita.(17)

Con respecto al tipo de evento adverso, en pediatría las caídas fueron los sucesos de mayor frecuencia, aspecto que coincide con lo encontrado en este estudio. El reporte de las caídas como un EA, es una situación común entre los niños por su condición de desarrollo (menor estabilidad al andar), o bien por su necesidad de exploración, que los coloca en una situación de mayor riesgo. En este estudio fue considerado como un EA, porque responde a la descripción del mismo, al ser un daño no intencionado, resultado desfavorable por acción u omisión durante la atención (ej. vigilancia del niño), y que puede ser evitado con el establecimiento de normas o procedimientos.

Como conclusión de este estudio en la ESE de Chile, se obtuvo que la identificación de los eventos adversos es fundamental para lograr una atención de calidad y es responsabilidad de cada integrante del equipo de salud velar por su cumplimiento. A su vez, dicha responsabilidad no solo debe limitarse a la notificación 
de estos sucesos, sino que también debe contribuir al conocimiento, desarrollando nuevas investigaciones en torno al tema. Ante lo expuesto, se hace fundamental la realización de futuros estudios, que permitan complementar la información necesaria y que contribuyan, fundamentalmente, a mejorar la atención que día a día se brinda a los pacientes más cuando estos son menores de edad.

En caldas en una ESE de primer nivel, estudio descriptivo de corte transversal, retrospectivo, que analizó la información obtenida de los eventos adversos registrados en los años 2007 a 2009. Como resultados se obtuvo que, se evaluaron 49 historias clínicas, correspondientes a 38 mujeres y 11 hombres. El 77,6\% eran mujeres. Similar a esta investigación siendo el género femenino prevalente en la presentación de eventos adversos. (18) Relativo a esta investigación llevada a cabo debido a que, el $55,6 \%$ de la población afectada con los accidentes en la atención son de sexo femenino.

El $71,1 \%$ de los E.A. que se presentaron en el sexo femenino eran prevenibles. Conocer la magnitud de las consecuencias de cada uno de estos accidentes, permitiría establecer la atención en la prestación del servicio adecuado y con calidad. Debido a que un evento adverso prevenible es el resultado no deseado asociado con un error en el suministro de la atención; esto se presenta porque no se utilizan correctamente los estándares del cuidado asistencial.(9) Según un estudio realizado por el profesor del Instituto de Investigaciones Clínicas de la Universidad Nacional de Colombia, Gaitán y por el grupo de Evaluación de Tecnologías y Políticas en Salud (2006), en Colombia la mortalidad asociada a los eventos adversos es cercana al 6\%. (19) Es por esto que, si los eventos adversos prevenibles se hubieran evitado, si se asumieran todos los más altos estándares de cuidado al paciente, la calidad de la prestación de los servicios aumentaría, como también la fidelidad de los clientes.

En los Estados Unidos, país donde se ha estudiado de manera más completa el problema de la seguridad de los servicios de salud, se ha estimado que al menos 44.000 pacientes fallecen en los hospitales anualmente como consecuencia de deficiencias en la atención a los usuarios, los cuales en su mayoría habrían podido ser prevenidos. La mayoría de estos eventos se relacionan con reacciones adversas a medicamentos, transfusiones inapropiadas, daños en cirugía, cirugías en sitios equivocados, caídas, fallas en la identificación del paciente y muertes, entre otros.(10)

Por otra parte, la investigación realizada en una ESE de Caldas, en cuanto a la afiliación al SGSSS la mayor proporción corresponde al régimen subsidiado con el $57,1 \%$, seguido del pobre no afiliado $(24,5 \%$, ) y el régimen contributivo $(10,2 \%)$; resultados similares a esta investigación debido a que el $64,3 \%$ de la población afectada tiene régimen subsidiado y/o vinculado. En el estudio de Gaitán-Duarte(10), el 44,0\% de los pacientes estaban afiliados a medicina prepagada, el $28 \%$ al régimen contributivo, el 21,5\% al régimen subsidiado y el $54 \%$ no tenía aseguramiento. En cuanto a la afiliación al SGSSS, este estudio mostró que la mayor prevalencia de eventos adversos se registró en pacientes afiliados al régimen subsidiado, con un $57,1 \%$, seguido del pobre no afiliado con un $24,5 \%$, y, por último, al régimen contributivo con un $10.2 \%$. Sin embargo, estos datos son poco relevantes, pues no se estudió toda la población y no se tuvo en cuenta el porcentaje de afiliación y contratación por régimen y entidad.(10)

Igualmente, el $71,2 \%$ de las complicaciones que se presentaron correspondían a pacientes afiliados al régimen subsidiado, y el $28,6 \%$ a pobres no afiliados. 10 Aunque los eventos registrados, en su gran mayoría, fueron eventos prevenibles, es muy relevante el número de eventos centinela presentados en los pacientes en la institución, los cuales estaban asociados a los fallos en el sistema de referencia y contrarreferencia de pacientes a un nivel de mayor complejidad que se refleja en la muerte por inatención.

La prestación de los servicios de salud muchas veces está determinada por el régimen que el paciente presenta, siendo el contributivo más privilegiado por la cancelación particular de las atenciones, presentándose entonces el subsidiado con más falencias o demoras en las asistencias de salud. Ante la evidencia de que personas desfavorecidas socialmente tienen peor situación de salud, la atención a las desigualdades han sido tema de discusión en la agenda política de la Organización Mundial de la Salud.(20)

Cabe anotar que la mayoría de la población de nuestro país pertenece a este sistema, debido a que es el mecanismo mediante el cual la población más pobre del país, y sin capacidad de pago, puede obtener los servicios de salud.(6) Debido a esto y dentro de las 
desigualdades en salud, se puede señalar el riesgo, la percepción de la salud, las diferencias en el diagnostico o tratamiento de patologías, la incidencia de la enfermedad, incapacidad o muerte, consecuencias socioeconómicas de las alteraciones de la salud, así mismo el comportamiento de la búsqueda de asistencia.

En cuanto a la relación entre el evento adverso y las barreras por las que se puede ocasionar, se observa que el $85,7 \%$ de la población presentan barreras humanas administrativas, puesto que fueron afectadas por las fallas en el cuidado directo. El análisis de estas barreras es una medida de control diseñada para prevenir el daño de elementos de valor o vulnerables. Las barreras humanas son las producidas por las personas y también por los factores que intervienen en su trabajo.

Para generar barreras de seguridad en la prestación de los servicios en salud, debe no solamente existir sino también ser funcional y positivo el reporte de los eventos adversos. El estudio Eventos adversos durante la atención de enfermería en unidades de cuidados intensivos, tiene similitud con la investigación llevada a cabo, en cuanto que, los factores de riesgo que inciden en la aparición de eventos adversos; donde la principal barrera es la humana - administrativa con una representación de 85,7\%.(21) Para este, las variables que intervienen en diferentes momentos del proceso de atención en salud, por sus condiciones específicas permiten establecer que la ocurrencia de un evento adverso no es unicausal donde las diferentes variables se mezclan y funcionan como piezas de un engranaje que forja un resultado funesto y perjudicial, donde todo actúa como un círculo vicioso permitiendo la aparición del efecto del "queso suizo".

De estas, sobre las que más se hace énfasis, son las que tienen que ver con el profesional; el equipo de trabajo, condiciones laborales; se enuncian los factores propios del paciente como factor de riesgo, siendo este el menos contemplado, lo que puede tener explicación en que se concibe al paciente crítico como aquel individuo que por procesos fisiopatológicos se encuentra inestable al borde de la muerte, que está indefenso y que necesita que se le satisfagan de manera parcial o total sus necesidades y que por lo mismo sería capaz de propiciar situaciones que le pongan a el mismo en riesgo(22). En cuanto a la relación entre el evento adverso y los factores se observa que el $70 \%$ de la población que presentan barreras en el paciente
- equipo tuvieron fallas en el cuidado directo

Tanto los elementos clínicos como los organizativos son influyentes para la exposición de un evento adverso; estas condiciones influencian la práctica y resultado de la atención en salud. A la cabeza de los factores contributivos, están los del paciente. En cualquier situación clínica, las condiciones de salud de este son un papel determinante sobre el proceso de atención y sus resultados. Otros factores del usuario son su personalidad, lenguaje, creencias religiosas y problemas psicológicos, todos los cuales pueden interferir la comunicación adecuada con los prestadores. La forma en que una determinada función se planea y la disponibilidad de guías y pruebas de laboratorio pueden, igualmente afectar la calidad de atención.(22)

Los factores del individuo (prestador) tales como conocimiento, experiencia, pericia, cansancio, sueño y salud, tanto física como mental, son condiciones que, dado el escenario propicio, pueden contribuir a que se cometan errores5. La atención de salud es cada día más compleja y sofisticada, lo que hace necesaria la participación de más de un individuo en el cuidado de cada paciente e imprescindible la adecuada coordinación como también comunicación entre ellos; en otras palabras, la atención de un paciente en la actualidad depende más de un equipo que de un individuo(5). Por este motivo, todo el personal de salud debe entender que sus acciones dependen de otros y condicionan las de alguien.

También, las decisiones y directrices organizacionales, originadas en los niveles gerencial y directivo de la institución, afectan directamente a los equipos de trabajo. Por otra parte, un evento adverso es aquel que genera daño al paciente, después de que éste ingresa a una IPS y además está más relacionado con el cuidado que con la misma enfermedad base.

Los eventos no prevenibles son aquellos que, a pesar de aplicar los mejores estándares de cuidado, no se pueden evitar. También son prevenibles los que se habrían podido evitar, si se hubieran utilizado todos los más altos estándares de cuidado. La investigación mostró que la incidencia de eventos adversos en los hospitales estudiados fue del 4.6\%; esta fue mayor en las especialidades quirúrgicas, con un $6.2 \%$, seguida por medicina interna, con cerca de $3.5 \%$; aproximadamente, el $60 \%$ de los eventos detectados eran prevenibles80. Este estudio halló que los eventos adversos 
en hospitales se dan más por fallas del sistema que por negligencia o falta de idoneidad del profesional de la salud; las causas estuvieron relacionadas con deficiencias de los procesos de provisión de servicios, problemas de racionalidad técnico-científica, problemas de comunicación entre los servicios hospitalarios e insuficiencia administrativo. Como resultado se obtuvo que, las instituciones participantes están desarrollando sus propios programas de seguridad del paciente.(22) Lo que al compararse con el presente estudio coincide debido a que se observa un porcentaje global del evento adversos del 4,2\%.

De igual forma, ya mencionado anteriormente en un Hospital Pediátrico de la ciudad de Santiago de Chile; se realizó estudio de carácter descriptivo, correlacional, retrospectivo: se revisaron 115 fichas clínicas, correspondientes a los ingresos entre los meses de enero del 2006 y julio de 2007, extrayéndose a través de una ficha de recolección de datos los eventos adversos notificados, entre otras variables. En el 15,7\% de los niños se notificó la ocurrencia de EA; en el 6,1\% se registró la ocurrencia de dos o más EA. Los EA notificados fueron: EA en administración de medicamentos, caídas y golpes. Se observó asociación entre la ocurrencia de EA y el número de días de hospitalización, jornada del día y mes de año. No se observó asociación con la edad del niño.

\section{CONCLUSIONES}

- Se encontró que los eventos adversos en el servicio de pediatría fueron más frecuentes en lactantes y preescolares de género femenino, ya que la mayoría de los pacientes que se encuentran en este servicio corresponden a este grupo poblacional.

- Los eventos adversos presentes en este servicio se atribuyen a causas relacionadas con el cuidado directo de los pacientes.

- Existen fallas en las barreras humanas y administrativas que predisponen de manera directa a la ocurrencia de eventos adversos en el servicio por lo cual es necesario intervenirlas.

- Son más comunes los eventos adversos en pacientes del régimen subsidiado ya que son estos pacientes los consultantes más frecuentes a esta institución hospitalaria, además de que en el caso de régimen contributivo cuentan con un espacio diseñado para la atención de estos pacientes con mayores recursos físicos que contribuyen a la no ocurrencia de eventos.

- La mayoría de los eventos adversos que ocurrieron en el periodo de tiempo evaluado tienen causas prevenibles. 


\section{BIBLIOGRAFÍA}

1. Ministerio de Salud y Protección Social. (2007).Guía práctica de preparación para la acreditación en salud. Recuperado el 18/11/2014, en: http://www.acreditacionensalud.org.co/userfiles/file/ Gu\%C3\%ADa\%20preparaci\%C3\%B3n\%20acreditaci\%C3\%B3n\%20MPS.pdf

2. Ministerio de Salud y Protección Social. (2012). Lineamientos para la implementación de política de seguridad del paciente en la República de Colombia 2012. Recuperado el 18/11/2014, en: http://saludcapital.gov.co/Publicaciones/Garantia\%20de\%20Calidad/Seguridad

3. Organización Mundial de Salud (OMS). (2009). Marco conceptual de la clasificación internacional para la seguridad del paciente. Recuperado el 18/11/2014, en: http://www.who.int/patientsafety/implementation/icps/icps_full_report_es.pdf

4. Ministerio de sanidad y política social (España). (2010). La seguridad en los cuidados del paciente hospitalizado proyecto SENECA. Recuperado el 18/11/2014, en: http://www.msssi.gob.es/organizacion/ sns/planCalidadSNS/pdf/ProyectoSENECA.pdf

5. Gaitán, L. M. y Garzón, A. (2010). Evaluación de la existencia de un protocolo sobre buenas prácticas para la seguridad del paciente e identificación de los factores de riesgo en la corporación IPS Tolima sede Interlaken y elaboración de un plan de mejoramiento para la prevención de eventos adversos. Universidad EAN. Ibagué, Colombia. Recuperado el 23/11/2014, en: http://repository.ean.edu.co/bitstream/handle/10882/525/GaitanLina2010.pdf?sequence=2

6. Ministerio de Salud y Protección Social. (2014). Observatorio de la calidad en la atención en salud de Colombia. Recuperado el 18/11/2014, en: http://www.minsalud.gov.co/Paginas/Observatorio-deCalidad-respondera-a-nuevo-enfoque-de-servicios-de-salud.aspx

7. Ministerio de Salud y Protección Social. (2008). Lineamientos para la implementación de la política de seguridad del paciente en la república de Colombia. Página 1. Recuperado el 18/11/2014, en: http://www. minsalud.gov.co/Documentos\%20y\%20Publicaciones/Lineamientos_para_Implementaci\%C3\%B3n_ de_Pol\%C3\%ADtica_de_Seguridad_del_Paciente.pdf

8. Requena, J., Miralles, J. J., Mollar, J. y Aranaz, J. M. (2011). Seguridad clínica de los pacientes durante la hospitalización en pediatría. Revista de calidad asistencial, 26(6). Recuperado el 06/01/2015, en: $\quad \mathrm{http}: / /$ apps.elsevier.es/watermark/ctl_servlet?_f=10\&pident_articulo=90038281\&pident_ usuario $=0 \&$ pcontactid $=\&$ pident_revista $=256 \&$ ty $=112 \&$ accion $=$ L\&origen $=$ zonadelectura $\& w e b=\mathrm{W}$

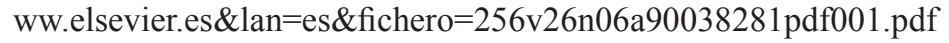

9. Bautista, L. M., Parada, D., Ballesteros, N., Rodríguez, J., Ornella, Z., Guampe, L. y Polentino, C. V. (2011). Frecuencia, evitabilidad y consecuencias de los eventos adversos. Revista ciencia y cuidado volumen 8(1), 75-84. Recuperado el 03/10/2014, en: http://dialnet.unirioja.es/servlet/ articulo? codigo $=3853523$

10. Gaitán-Duarte, H., Eslava-Schmalbach, J., Rodríguez-Malagon, N., Forero-Supelano, V., SantofimioSierra, D. y Altahona, H. (2006). Incidencia y evitabilidad de eventos adversos en pacientes hospitalizados en tres instituciones hospitalarias en Colombia. Recuperado el 02/01/2015, en: http://www. scielosp.org/pdf/rsap/v10n2/v10n2a02.pdf

11. Ministerio de Salud y Protección Social. (2009). Evaluación de la frecuencia de eventos adversos y monitoreo de aspectos claves relacionados con la seguridad del paciente. Recuperado el 18/11/2014, en: http://www.minsalud.gov.co/sites/rid/Lists/BibliotecaDigital/RIDE/DE/CA/Evaluar_frecuencia eventos_adversos.pdf\#search $=$ eventos\%2520adversos

12. Álvarez, C. A. (2009). Comprometidos con el conocimiento y el control de las infecciones. EDITORIAL. Infectio, 13(2). Recuperado el 09/10/2014, en: http://www.scielo.org.co/pdf/inf/v13n2/ v13n2a01.pdf

13. Luengas. S. (2008). Modelo para gestionar la seguridad del paciente en las instituciones de salud. Viasalud, (43), 8-15. Recuperado el 12/12/2014, en: http://www.cgh.org.co/imagenes/calidadycultura.pdf

14. Cárdenas, N., (2010). Modelo de calidad en la gestión de salud con énfasis en la seguridad del paciente, para la terapia endovascular de aneurismas intracerebrales en un servicio de neurointervencionismo. Recuperado el 11/11/2014, en: http://repositorio.utp.edu.co/dspace/bitstream/11059/1479/1/658562C 
$266 \mathrm{~m} . \mathrm{pdf}$

15. Fernández, N. (2004). Los eventos adversos y la calidad de atención. Estrategias para mejorar la seguridad de los pacientes pediátricos. Archivos Argentinos de Pediatría, 102(5), 402-410. Recuperado el 02/12/2014, en: http://www.seguridaddelpacienteyenfermero.com/docs/articulos/articulo_03.pdf

16. Riquelme, G y Ourcilleón, A. (2013). Descripción de eventos adversos en un hospital pediátrico de la ciudad de Santiago, Chile. Enfermería global, 12(1). Recuperado el 29/11/2014, en: http://revistas. um.es/eglobal/article/view/158551/144061

17. Moreno, S., Gutiérrez, C, Osorio, J. y Segura, A. M. (2010). Efectos adversos asociados a la vacunación PAI en niños menores de 2 años en Medellín reportados entre 2008-2009. Noviembre 16 de 2010. Recuperado el 18/11/2014, en: http://revistas.ces.edu.co/index.php/ces_salud_publica/article/ view/1307

18. Otálvaro, A. M., Valencia, M. A. y Cardona, D. (2011). Caracterización de Eventos Adversos en una E.S.E de Primer Nivel en Caldas 2007-2009. Hacia la Promoción de la Salud, 16(1), 87-98. Recuperado el 02/02/2015, en: http://promocionsalud.ucaldas.edu.co/downloads/Revista16(1)_7.pdf

19. Páez, M. (s.f.). Eventos adversos un desafío para la medicina. Universidad Nacional de Colombia. Bogotá. Recuperado el 02/02/2015, en: http://historico.unperiodico.unal.edu.co/ediciones/110/10.html

20. Castro, M. G. (2009). Evaluación de la utilización adecuada del control prenatal en bogotá con base en la encuesta nacional de salud 2007. Universidad Nacional de Colombia. Bogotá, Colimba. Recuperado el 11/11/2014, en: http://www.bdigital.unal.edu.co/2615/1/597350.2009.pdf

21. Bernal, D. C. y Garzón, N. (2008). Eventos adversos durante la atención de enfermería en unidades de cuidados intensivos. Pontifica Universidad Javeriana. Bogotá, Colombia. Recuperado el 03/10/2014, en: http://www.javeriana.edu.co/biblos/tesis/enfermeria/tesis44.pdf

22. Sarabia, O. (2007). Seguridad del paciente en la atención médica. Foro: "nuevos rumbos de las políticas de salud en México". Recuperado el 29/11/2014, en: https:/www.google.com.co/url?sa=t\&r $\mathrm{ct}=\mathrm{j} \& \mathrm{q}=\& \mathrm{esrc}=\mathrm{s} \&$ source $=$ web\& $\mathrm{cd}=2 \& \mathrm{cad}=\mathrm{rja} \& u a c t=8 \& \mathrm{ved}=0 \mathrm{CCIQFjAB} \&$ url $=\mathrm{http} \% 3 \mathrm{~A} \% 2 \mathrm{~F} \% 2 \mathrm{~F}$ www3.diputados.gob.mx $\% 2$ Fcamara $\% 2$ Fcontent $\% 2$ Fdownload $\% 2 F 115502 \% 2 F 312674 \% 2 F f i l e \% 2 F$ Seguridad_Paciente_Dra_Sarabia.pdf\&ei=QmX4VPaGI8W9ggSY1ILIAg\&usg=AFQjCNHwLXG_ EYunvYq3U5Kcjg_d8JNY9A\&bvm=bv.87519884,d.eXY 